\title{
What has aviation got to do with dentistry?
}

Dental Showcase is challenging visitors to learn not only from their peers, but also from those outside of the industry. In a captivating lecture, entitled 'Lessons from the Cockpit', Steve Hawkins, BA's Chief

Training Pilot, will compare aviation and dentistry.

Surprisingly there are a lot of similarities between the two. Risk is inherent in both - for the provider (pilot/dental professional) and the customer (passenger/ patient). Similarly, dentistry and aviation are overseen by regulatory bodies, who set the standards, risk management, training and ongoing assessment of competence. Safety is key to both industries; however, Steve will explore how attitudes to the reporting cultures of the two industries may differ.

The aviation industry actively encourages mistakes to be reported, without fear of recrimination. Readers will be all too familiar with the growing number of cases brought against dentists by the General Dental Council. Clearly there is some disparity between the open reporting culture of the airline industry and the sometimes 'closed loop logic' of healthcare.

Steve will be just one of the captivating speakers assembled as part of the Dental

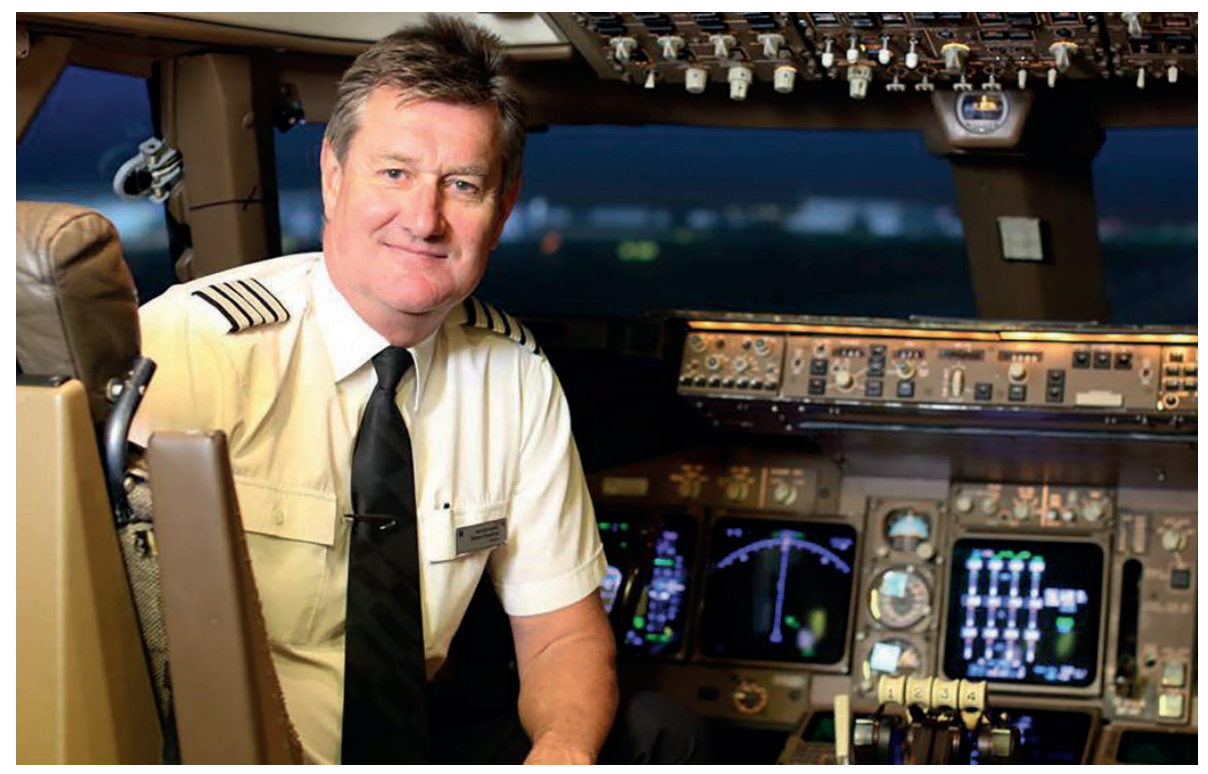

Update Study day on Thursday 4 October, hosted by Professor Trevor Burke, who will be giving a lecture entitled 'Minimising Failure with Direct Restorations and Crowns'. John Milne, Head of the CQC, will also present an engaging and potentially contentious session on regulatory matters whilst Tara Renton, will look at specific risks associated with oral surgery
A full programme of lectures continues Friday and Saturday, with presentations by Louis MacKenzie on anterior composites. Paul Bachelor will explore the topic of dementia-friendly dentistry and Bob Cummings will explain some of the HMRC challenges associates face.

To register, or for more information, visit dentalshowcase.com.

\section{More than just a long-lasting post}

Improved dentin preservation and better aesthetics are two of the convincing advantages of VDW's DT Posts. These are resulting from VDW's Double Taper Shape design and quartz fibre technology: the key to a long-lasting endodontic treatment success.

For endodontically treated teeth with more than one missing dentin wall the placement of a post to maintain the coronal structure is strongly suggested. To place it properly, it is key to retain as much dentin as possible while preparing the root canal beforehand. VDW's DT Posts with Double Taper Shape preserve more dentin as the two-stage design corresponds optimally to the morphology of the prepared root canal. Thus, the dentist avoids unnecessary dentin removal to fit in the post.

The DT Posts' break-resistant quartz fibre material has advantageous

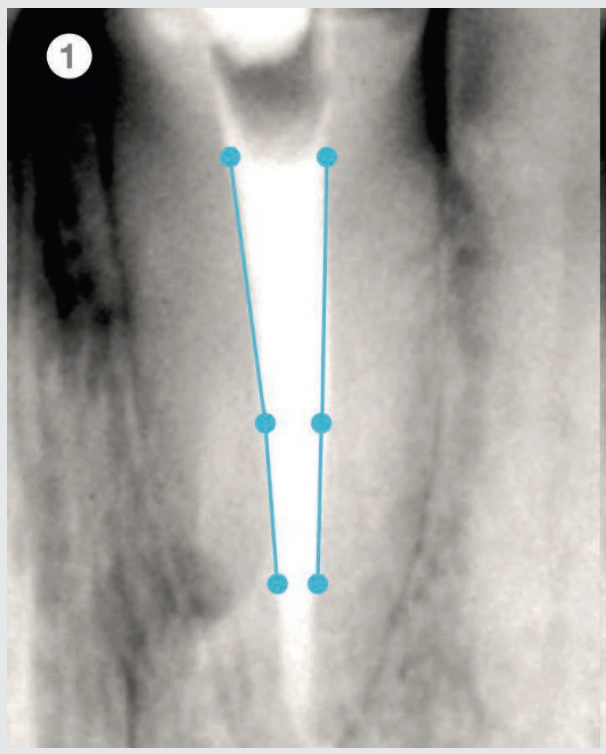

mechanical characteristics. Its low modulus of elasticity distributes chewing forces correctly and minimises the risk for root fractures. Thanks to the quartz fibre

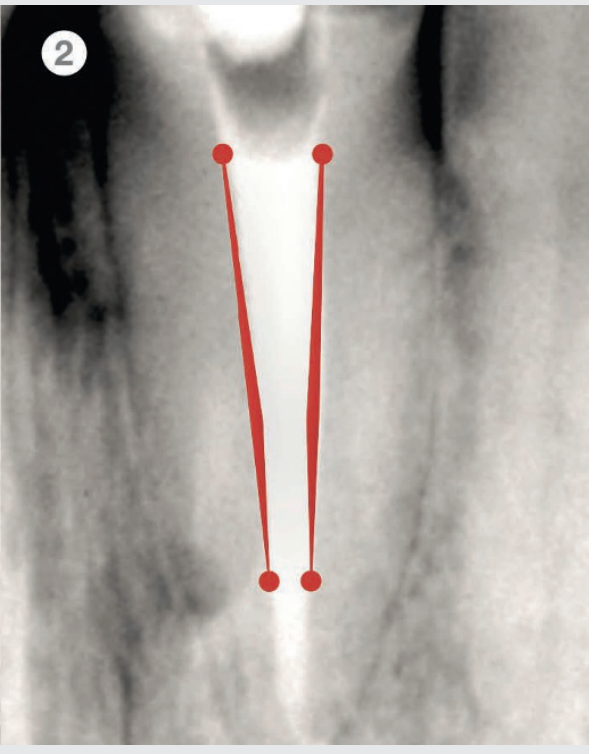

material's translucency properties the patient benefits from better aesthetics.

Read more at https://www.vdw-dental. com/en/products/post-endo/ 